\title{
Functional Modeling and General Collective Intelligence as the Basis for Pervasive Healthcare
}

\author{
Andy E. Williams ${ }^{1, *}$ \\ ${ }^{1}$ Nobeah Foundation, Nairobi, Kenya
}

\begin{abstract}
General Collective Intelligence has the potential to combine individuals into a single collective collective intelligence with general problem-solving ability (intelligence) that might be exponentially greater than that of any individual. In every software domain, including health and wellness, General Collective Intelligence and functional modeling have the potential to enable the definition of pervasive cognitive computing applications and platforms. In such cognitive apps, intelligent agents might provide services to the user that optimize their outcomes by independently executing functional operations in each software domain on whatever software best implements those operations, and independently incorporating any possible data available to the user in the best way available. And at the same time in such cognitive computing platforms, a GCI might orchestrate the process of gathering data from all such individual uses in order to optimize collective outcomes such as significantly increasing healthcare and wellness. And these models of individual and collective cognition suggest that such optimization might not be reliably achievable otherwise. For both of these cognitive computing approaches functional modeling is required to provide a universal mechanism for representing data and processes. Therefore, to achieve significantly increased healthcare and wellness outcomes both functional modeling and GCI might be required. Functional modeling has the potential to overcome the lack of consistency in type and format of data gathered and the lack of a mechanism for universally comparing and combining that data. This paper explores why functional modeling might not only be of critical importance to pervasive healthcare, but why it also might be critical to significantly improving capacity to diagnose and to make interventions.
\end{abstract}

Keywords: General Collective Intelligence, Human-Centric Functional Modelling, Artificial General Intelligence, healthcare

\section{Background}

The covid-19 pandemic has revealed that medical research is not nearly as objective as once thought. Anecdotally, personal inclination towards one set of cognitive biases or another appears to clearly have an impact on both the medical research one might believe to be credible, and the conclusions that medical researchers come to. In a question as simple as whether or not wearing masks reduces the spread of the virus, studies give conflicting results [1] potentially due to such biases [2]. And studies that attempt to combine those results might merely obfuscate that bias through committing the fatal flaw of combining fundamentally incompatible datasets (i.e. trying to average an apple with an orange) which this paper suggests renders them as useless. By defining a functional model as universal representation of the human system in which truth has a well-defined pattern, this paper explores how such weaknesses can be identified and eliminated in order to define cognitive apps and platforms with the capacity to provide pervasive access to health and wellness services. And this paper explores how this

"Corresponding author. Email: awilliams@nobeahfoundation.org 
cognitive computing might significantly increase any targeted outcome in health and wellness care.

Human Centric Functional Modeling (HCFM) [3] represents any dynamical system with repeatable states in terms of the minimal set of reversible functions required to enter those states. The states accessed through these functions form a "functional state space" through which the system moves. By modeling all of the systems functions, this approach can be used to represent all the behavior of complex systems even where the mechanisms by which that functionality are implemented are unknown. By using this approach to define a Functional Modeling Framework (FMF) [4] for modeling the human system has been defined. This framework has been used to define what is believed to be the first model of artificial cognition (Artificial General Intelligence or AGI [5]) with the potential capacity for human-like general problem-solving ability. And it has been used to define the first model of collective cognition (General Collective Intelligence or GCI [6], [7]) with the potential for exponentially increased intelligence.

\section{Introduction}

GCI introduces the potential to exponentially increase general problem-solving ability through creating the potential to exponentially increase three capacities. These are the capacity to navigate complexity in collective reasoning processes, the capacity to scale the number of collective reasoning processes executed and the number of participants and resources involved, and the capacity to align cooperation between those processes in order to create sufficient value that the cooperation becomes self-sustaining. In any problem domain in which impact on outcomes is limited by general problem-solving ability, rather than being limited by physical resources or other limits, an exponential increase in general problemsolving ability can drive an exponential increase in impact on targeted outcomes.

Therefore, wherever outcomes in the health or wellness care domain can be increased through these three capacities without physical limits, GCI has the potential to exponentially increase health and wellness care outcomes. In the case that such physical limits do exist, GCI might still significantly increase such outcomes. Consider all processes involved in health and wellness care, from the business processes of health care providers, to the business processes of health care insurers, as well as others. Representing each process using a swim lane diagram, then the activities conducted by each participant in the process will be confined to each lane.

\section{Outcomes of Collective Processes}

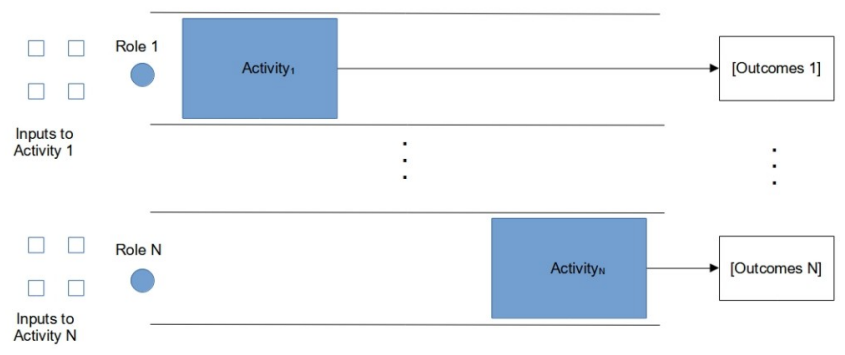

Figure 1. Swim lane diagram representing activities and participants in any process related to health or wellness care.

One way efficiency in achieving outcomes might be increased is to scale those processes over more, fewer, or potentially different participants wherever doing so makes processing more efficient. This includes automating some processes so they might be performed at greater speed and scale by fewer participants with more processing resources, or so they might be performed at greater speed and scale by more participants with less processing resources. Having general problem-solving ability, a GCI as an adaptive collective problem-solving system might be applied to adapt such processes in this way.

As mentioned, wherever outcomes in health and wellness care can be increased through the three capacities provided by GCI (capacity for process complexity, capacity for number of processes, and capacity for processes to be self-sustaining), then GCI might be used to significantly increase outcomes. Using GCI to increase the outcomes of 
healthcare business processes requires health and wellness care processes also have the capacity to increase outcomes through increasing their complexity, through increasing the number of processes and participants, and through increasing capacity to make processes self-sustaining. Scaling processes over different participants and their resources requires a common model of healthcare data so that it is portable to different health care providers. A significant degree of portability might be achieved by defining a common canonical model of healthcare data and defining a translation infrastructure so that each health care provider's data can be translated to and from their internal data formats to that canonical data format.

Using GCI to increase the outcomes of medical interventions again requires the capacity to increase the complexity of interventions, the capacity to increase the number of interventions and participants, and the capacity to make interventions self-sustaining. Scaling interventions in this way requires a common model of the human system so that the state of the human system and the impact of interventions on that state is portable across all different health care practitioners. Any such model must provide completely self-contained descriptions, therefore it must be a semantic model. This model must also provide a complete representation of the functions of the human system, even where the mechanisms by which those functions are implemented are currently unknown. Therefore it must also be a functional model.

Implementing GCI might then begin with a canonical model of health care data, and then evolve into implementing a functional model of the human system.

Two applications of GCI to the design of software platforms related to healthcare have been proposed to date. One is an Electronic Health Record (EHR). The other is a Provider-based Health Insurance platform. An assessment of the potential combined savings to the health system due to collectively intelligent cooperation suggests that potential savings to be sufficient to significantly increase access to affordable health care, and to significantly increase quality of health care at a given cost level. For private health care systems the cost savings might be sufficient to pay for universal healthcare for those without coverage. For public health care systems a significant increase in quality of health care at a given cost might address issues with decreased service levels.

While such platforms might begin with a canonical data model, a semantic data model based on a functional model of the human system has profound positive long-term implications. The functional state spaces representing the human system might be used as tools for making clinical observations in order to overcome the problem of lack of consistency and comparability in medical data. For example every plant on earth is a potential medicine in that it has some effect on the human system. However observing those impacts across all studies in a coherent and automated way is difficult with the unstructured and incomplete data generally available outside of a single targeted research project. Defining the human system in terms of unambiguous functional state spaces, and defining medicines and other interventions as transforms in those state spaces is proposed to enable observations made in different systems of medicine to be comparable in common terms, so they can be combined into a single database in which a medical study to deduce the impact of any intervention is simply a slice of the data. Such a data representation is likely to become increasingly important in the long term as health and wellness care platforms become more pervasive.

\section{Related Work}

Though others have described the concept of a general collective intelligence factor (c factor) [8], though at least one other has defined a model for a general collective intelligence factor [9], and though others have described the concept of a collective super intelligence [10], to the author's knowledge the author's own work is the only model that defines the specific mechanisms required for a General Collective Intelligence or 
GCI with the potential for exponentially greater general problem-solving ability (intelligence) than any individual. Because of this, and because this model is so new, all of the existing literature surrounding GCI refers to the author's own published work, or pre-prints of the author's unpublished work that are still under review.

\section{Purpose, Research Questions, and Approach}

The purpose of this paper was to demonstrate the capacity of General Collective Intelligence to significantly increase health and wellness care outcomes through using the individualization of health and wellness care services to increase the three capacities through which GCI can significantly increase any targeted outcome. And to demonstrate the capacity of General Collective Intelligence to target the right health and wellness care outcomes pervasively in order to maximize collective well-being through maximizing the well-being of each individual.

The research questions addressed in this paper and the method with which they were approached are described below:

\section{Section 5: Model}

5.1. Defining a functional model for the human system.

5.2. Defining truth in the functional model of the human system.

\section{Section 6. Method}

6.1. An analysis is performed to assess how functional models of healthcare and wellness interventions might be individualized.

6.2. An analysis is performed to assess whether and how the truth of conclusions driving individualized interventions might be determined.

6.3. An analysis is performed to assess whether interventions might be individually and collectively optimized according to such conclusions.

Section 7. Findings

Section 8. Research Limitations

Section 9. Practical Implications

Section 10. Conclusions

\section{Model}

Aspects of the functional modeling approach, and aspects of General Collective Intelligence that are relevant to the research questions are described below.

\subsection{Functional Modelling of the Human System}

A system like the body that has repeatable functions can perform those functions again and again. In other words the existence of those functions is stable. A system like an egg that does not have repeatable functions might only perform a function once, like the egg breaking. For any system with repeatable functions, including the human body, functional modeling can potentially be used to define a functional state space with the capacity to represent the system. Each functional state space might have some general properties in common that decouple the meaning of the state from any functional component of the system so that any functional component of the system can be optimized according to the same definition of state. In the conceptual space of the cognitive system this means that concepts must be defined in a way that is independent of any of the functional components implementing those concepts so that the reasoning implemented by those functional components can be optimized collectively by all functional components. In the collective conceptual space of a GCI this means that concepts must be defined in a way that is independent of any individual or organization participating in the collective reasoning, so that the collective reasoning implemented by that GCI might be optimized collectively by all participants.

The requirements for decoupling descriptions in functional state space are spread across a number of domains of collectively intelligent cooperation. For example, each region in functional state space must be described through its relationships with other regions (peer-to-peer). Each relationship must be described through relationships that are observable by the user rather than theorized (usercentric). The entire network of relationships together must collaborate to define a functional 
state space (massively collaborative). And that functional state space must not have any centralized store of meaning that can be aligned with any interests other than optimizing outcomes (decentralized). These similarities between functional state spaces might arise from their common purpose of capturing the functional dynamics of some system in a given domain. Lessons learned in implementing any one functional state space, such as a functional state space defined for the domain of physical interactions in physics, might then potentially be generalized and reused in another domain such as the human system.

As mentioned, the Functional Modeling Framework (FMF) [4] defines a framework for modeling the human system including the body, emotions, cognition, and consciousness. The models of cognition and consciousness have already been detailed elsewhere [11], [12]. Within this same framework ongoing work continues to elaborate a functional model for the body [13].

\subsection{Using Functional State Space to Assess the Truth of Conclusions Regarding Interventions}

Part of the usefulness of leveraging functional modeling to define functional state spaces for the entire human system is that as in every other case, functional modeling has the potential to determine truth (what functions to be true) for the human system more unambiguously than possible today [14]. This has great implications in medicine.

Human beings are capable of two types of reasoning, type 1 (intuitive or "fast") reasoning, and type 2 (rational methodical or "slow") reasoning [15]. And in the reasoning they express human beings can be anywhere on the spectrum from intentionally truthful, intentionally untruthful, or somewhere in between. Type 1 reasoning (intuition) is based on recognition of past patterns and is used in a number of circumstances, one of which is navigating problems that are too complex to be broken down into any methodical reasoning process. Type 1 reasoning intentionally or unintentionally ignores information that is inconsistent with its conclusion. For people who don't use type 1 reasoning on a given topic, anecdotally type 1 reasoning might be conflated with dishonesty, which intentionally distracts from information that is inconsistent with the conclusion it is trying to convey. Both intuitive reasoning and deception reasoning can result in essentially separate conceptual spaces for the facts used, and for the facts ignored. In other words, with this reasoning type, if the reasoning required to see the truth is contrary to an individual's intuition it exists in a separate conceptual space and individuals are not capable of seeing the truth. Truth cannot be discerned if it exists in a separate conceptual space from what one recognizes as the facts.

Type 2 (rational methodical) reasoning is based on evaluation of some reasoning logic or "equation" and is used where such reasoning exists and is known. Type 2 reasoning intentionally or unintentionally includes information that is irrelevant to its conclusion. For people who don't use type 2 reasoning on a given topic, anecdotally type 2 reasoning might be conflated with intentionally giving in to delusion, and therefore intentionally obfuscating reasoning with information that should be irrelevant to whatever conclusion that reasoning is trying to convey. Both rational methodical reasoning and delusion can result in systemic errors in reasoning that make the truth impossible to discern.

Assume that in a functional model any sickness somehow forms a well-defined pattern in that functional state space. If so, then whether one uses homeopathy, western medicine, or traditional Chinese herbs, the impact of those treatments should be more clear than currently achievable through mere written case histories which might provide incomplete information from the perspective of any given treatment.

As an of the usefulness of a complete semantic model of the body that is capable of storing and communicating all understanding about the body rather that storing and communicating information about the body, consider the example of comparing treatments using Chinese traditional 
medicine to treatments using western medicine. The treatment in Chinese traditional medicine might depend on one's "Qi", data the western case history wouldn't catch. Functional modeling of the human system provides a way for medicine to catch all data. Functional modeling in every other area is meant to do the same, as opposed to restricting data to a type 1 or type 2 reasoning based narrative. The point is not just to develop a map for familiar territory, but to develop a compass for unfamiliar ground ... not just to learn which facts are true or not true within one's limited context, but instead to learn what the truth functions to achieve so that one can recognize truth in the unfamiliar facts of any context.

\section{Visualizing Medical Interventions in the Functiona} State Space of the Human System

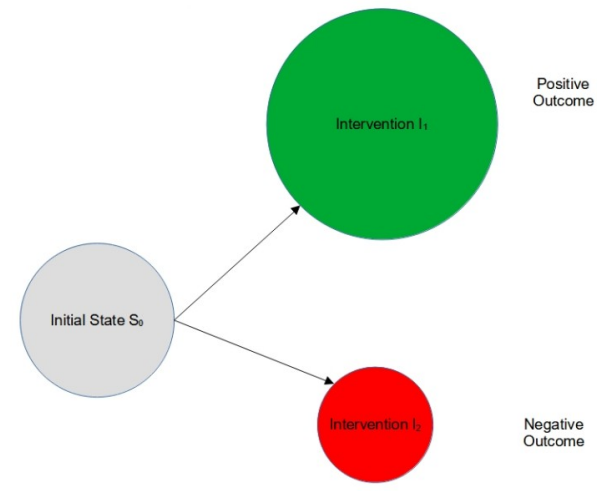

Figure 1. In the functional state space of the human system states are bounded regions and medical interventions move those regions.

Every patient has a different risk profile and other criteria defining their optimal medical or wellness intervention depending, on factors such age, or other complicating illnesses. Currently there is no single framework that enables a medical profile to be constructed for a patient, and that enables all possible combinations of all interventions to be represented in a uniform way so they can be compared and the optimal set of interventions selected in an automated way. There is also no framework that enables all medical services to be compared and the optimal ones selected in the same way. With the complexity of interventions and medical services that might potentially be analyzed, and with this comparative inability to automate that analysis, it is easy to obfuscate the truth by focusing on some factors to the neglect of others.

In order for such comparison to be possible, a common functional representation of the state of the human system and the impact of interventions must be constructed. Other work has detailed the definition of a human state space in which each point represents a functional state potentially defined by properties such as those below:

Table 1. Proposed properties of the functional state space of the human system.

\begin{tabular}{|l|l|}
\hline \multicolumn{1}{|c|}{ Property } & \multicolumn{1}{c|}{ Description } \\
\hline $\begin{array}{l}\text { [Conscious|Cognitive } \mid \\
\text { Emotional|Physical] } \\
\text { Well-Being }\end{array}$ & $\begin{array}{l}\text { Fitness to execute all } \\
\text { [Consciousness| } \\
\text { Cognitive|Emotional| } \\
\text { Physical] Functions }\end{array}$ \\
\hline $\begin{array}{l}\text { [Conscious|Cognitive } \mid \\
\text { Emotional|Physical] } \\
\text { Well-Being Momentum }\end{array}$ & $\begin{array}{l}\text { Change in well-being } \\
\text { over time }\end{array}$ \\
\hline $\begin{array}{l}\text { [Conscious|Cognitive| } \\
\text { Emotional|Physical] } \\
\text { Well-Being Acceleration }\end{array}$ & $\begin{array}{l}\text { Acceleration in well- } \\
\text { being over time }\end{array}$ \\
\hline $\begin{array}{l}\text { [Conscious|Cognitive } \mid \\
\text { Emotional|Physical] } \\
\text { Well-Being Force }\end{array}$ & $\begin{array}{l}\text { Well-Being * Well- } \\
\text { Being Acceleration }\end{array}$ \\
\hline
\end{tabular}

In such a functional state space the health of an individual is a bounded region in that space. The impact of an intervention is to move that region to a different area. Just as laws in physical space govern how matter in one area of space can transition to another area, the state of an individual patient will obey some "physics" in that functional state space that will determine whether the state of the patient might transform to another state, what paths (interventions) might facilitate that transformation, and how quickly that transformation might occur through each of those paths. As opposed to a single diagnostic method that captures data in a way that might not be comparable with the data captured by another diagnostic method, all diagnostic methods might 
produce different motions in such a functional state space. Those motions might be directly comparable. And if any motion predicted by any model of an intervention is "unphysical" in that it doesn't reflect actual observations, then that unphysical behavior might be used to identify where the model of the intervention (i.e. the "physics" of the functional state space) is incomplete or inconsistent. For example, broken bones don't spontaneously heal in seconds. And stage four cancer doesn't instantaneously disappear. If they do, then the models defining the "physics" with which the human health moves within the human state space are incomplete or inconsistent. In the exact same way that models in physics are compared against actual observations to confirm the correctness of, or to identify the holes in physical theories.

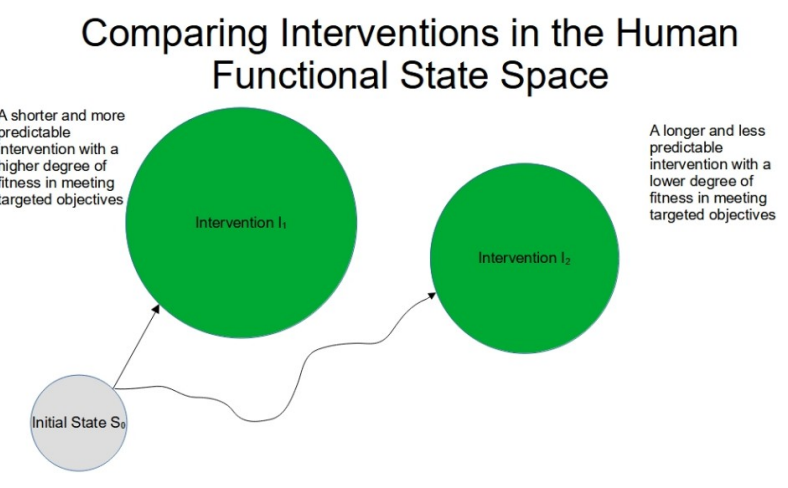

Figure 2. Interventions might be compared by their fitness in taking the patient to a target region

in the functional state space representing the human system. Shorter and more predictable paths are expected to be more fit.

Being potentially capable of representing observations gained from any diagnostic method, such a functional state space must have the capacity to represent any analysis metrics.

Figure 3. A functional state space defined for the human system must accommodate all analysis metrics.

Having defined a universal mechanism for comparing the fitness of any analysis method for any purpose related to the human state space,

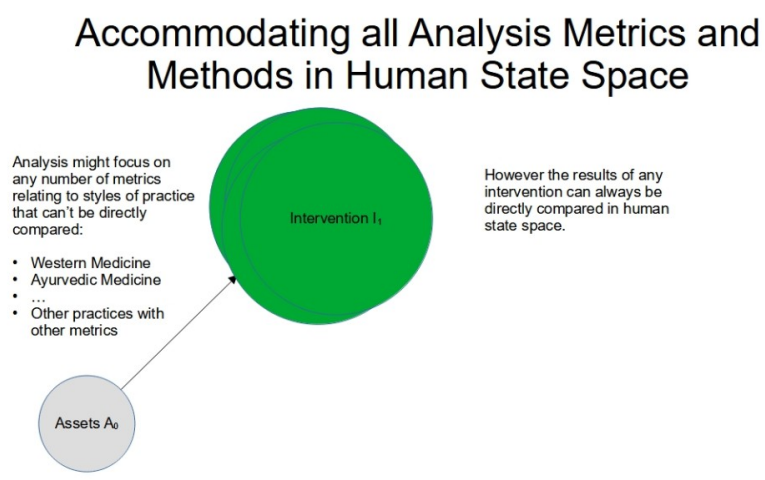

whether for assessing the state of the patient, or for assessing the relative fitness of a given intervention in changing that state, then a method for assessing truth in terms of whether behaviour predicted by an understanding of an intervention is "unphysical", can be defined.

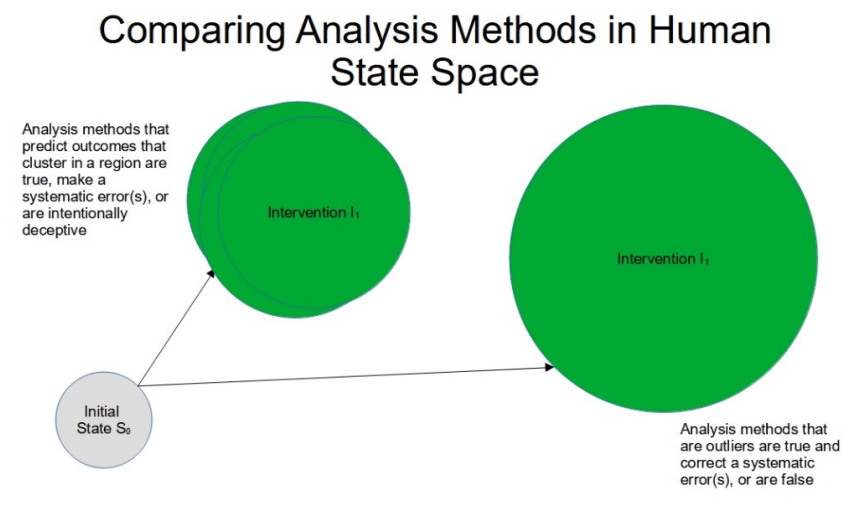

Figure 4. The fitness of different analysis methods might be compared.

In creating an analysis framework in which AGI might be used to automate research, and in which GCI might be used to coordinate all such research, such a framework creates the potential to significantly increase medical research capacity.

\section{Method}

The research question regarding how GCI might provide the basis for persistent health care was addressed using the methods summarized in table 2.

Table 2. Research question and method. 


\begin{tabular}{ll}
\hline Research Question & Method \\
\hline Doe General & 1. Assess how \\
Collective & functional models of \\
Intelligence have the & healthcare and \\
capacity to both & wellness diagnosis or \\
optimize targeted & interventions might \\
health care & be individualized. \\
outcomes, as well as & 2. Assess whether \\
the capacity to target & the truth of \\
the right outcomes in & individualized \\
order to maximize & diagnoses or \\
collective health and & interventions might \\
wellness? & be determined. \\
& $\begin{array}{l}\text { 3. Assess whether } \\
\text { interventions might } \\
\text { be individually } \\
\end{array}$ \\
& $\begin{array}{l}\text { optimized according } \\
\text { to those } \\
\text { requirements. }\end{array}$ \\
\hline
\end{tabular}

\subsection{Individualization of Interventions}

By defining a canonical model of the human system in terms of a large but closed set of properties, diagnostic methods might be defined to accept those properties as inputs, health care conditions might be defined in terms of those properties, and interventions might be defined in terms of those conditions. Results of those interventions might be defined in terms of those properties again. In the same way that regression testing in software associates a set of tests with each functionality, so that all potential errors in that functionality might be tested for whenever a change is made, batteries of diagnostic tests might be defined to test each relevant function after each stage of intervention.

By defining as many categories of profiles as required to characterize users of different age, existing conditions, and other properties related to health, and by using AI to detect patterns in the correlations between different values of different properties in the canonical model related to health, to detect patterns in the correlations between different diagnostic methods most likely to be most fit in observing health conditions or state of wellness in each profile, and to detect patterns in the correlations between interventions that are are most likely to be most fit in achieving targeted outcomes, AI might be used to effectively combine all healthcare into a single database that a pervasive health application or platform might leverage in customizing interventions in ways that significantly improve outcomes in whatever area it targets.

However, a canonical data model is by necessity limited by the fact that it makes assumptions regarding what the key properties defining the state of the human system are. Wherever these assumptions are incorrect or incomplete, and wherever they are inconsistent with some other data model, this approach can potentially fail in its capacity to represent the state of the human system, in its capacity to model diagnostic methods that are inconsistent with it, and in its capacity to model interventions that are inconsistent. This results in dimensions of health or wellness care services in the areas of diagnostics, or interventions that might be outside of the capacity of such a system to customize in order to optimize individual patient outcomes.

However, as described in more detail elsewhere [16] by defining the state of health and wellness of an individual as a region in a human state space, AGI might be used to automate the customization of diagnostic or intervention services for each individual across all possible implementations of those services, and GCI might be used to automate the customization of those services across all individuals and all practitioners. This customization can be performed in a way that acknowledges each individual's cognitive bias where that bias might determine which services the individual finds to be of value [17].

\subsection{Truth of Diagnosis or Interventions}

The number of possible diagnostic procedures, or interventions, continue to grow as medical science advances. But reliably converging on the truth of which diagnostic procedure or intervention is more fit in achieving targeted outcomes for each individual patient is still sometimes outside of current capacity. Conventional written health care 
data can reliably convey information, but cannot reliably convey an understanding or interpretation of that information. Health care data communicated using a canonical data model might more reliably convey an understanding, but that understanding has limitations because canonical data models are incomplete representations that cannot represent all possible data. In addition, canonical data models require learning the models themselves. A functional and semantic model of the human system however that is human-centric and therefore intuitive might reliably convey understanding. Creating the capacity to communicate an understanding rather than just information that must be interpreted is an important part of creating the capacity to assess the truth of what is being communicated. And since truth has a well-defined pattern in functional state space, defining a functional state space for the human system (the human state space) is important in determining the truth of any diagnosis or intervention.

\subsection{Optimization of Interventions}

As described in more detail elsewhere [16] by defining the state of health and wellness of an individual as a region in a human state space, AGI might be used to optimize diagnostic or intervention services across all possible implementations of those services, and GCI might be used to optimize those services across all individuals and all practitioners, in order to provide the capacity to significantly increase outcomes.

\section{Findings}

Text.

Table 3. Research Findings.

\begin{tabular}{ll}
\hline Research Question & Findings \\
\hline 1. Can functional state & The answer \\
spaces defined within & appears to be yes \\
a Human-Centric & pending \\
Functional Modelling & confirmation by an \\
\hline
\end{tabular}

\begin{tabular}{l}
\hline $\begin{array}{l}\text { approach be used to } \\
\text { define the }\end{array}$ \\
individualization \\
required to optimize a \\
product or service for \\
any individual user or \\
group, and can AGI \\
and GCI be used to \\
automate the \\
customization of those \\
products and services \\
for that individual or \\
to groups of \\
individuals?
\end{tabular}

\section{Research Limitations}

The functional model of the human state space has only been mentioned at a high level in this paper. It is outside of the scope of this paper to provide a detailed description of the functional models of the human system that are required to achieve mass individualization of healthcare and wellness services. A major effort to elaborate this functional model is required in order to achieve this individual customization. All functional state spaces are expected to have some common abstract properties. As the model of the conceptual space of the cognitive system, as well as models of other functional state spaces continue to be elaborated, this commonality should enable that effort to benefit the understanding of the functional state space for the human system, and all other functional states spaces as well.

\section{Practical Implications}

Virtually any impact can be delivered through a product or service. Increased access to more affordable healthcare, for example, can be achieved through more cost effective healthcare services. And local value chains with powerful competitive advantage can be designed to support virtually any product or service, including those healthcare services.

GCI based collectively intelligent cooperation has the potential to align cooperation within a network 
of project so that cooperation creates sufficient value for all those projects to be sustainably selffunding [19]. Phase II of a proposed Collective Intelligence based Program to Accelerate Achievement of the Sustainable Development Goals (CIPAA-SDGs) aims to deploy projects solutions that have the potential for remarkable savings in healthcare. Proposed health care policies of any country might be deeply politicized. For example, in the US the support for Trump care, or Obama care might run along political lines. However collectively intelligent solutions are potentially apolitical, and if GCI does in fact have the potential to exponentially increase ability to impact any general problem, GCI based health care might have the potential to save enough of the $\$ 7$ trillion global budget in healthcare for the savings to pay for universal healthcare coverage globally for those without.

One proposed GCI based health care solution is provider based insurance. Health insurance, as well as the cost of processing claims through the differing claims systems of multiple health care insurance providers, by some estimates accounts for up to $50 \%$ of the cost of health care in nations such as the US where private health insurance is prevalent. This $50 \%$ consists of the operational costs of the insurer, their profit margin, and the administrative costs of the healthcare provider, that is, the cost to doctors and hospitals of processing insurance claims. In other words, if the health care insurance industry is responsible for up to $50 \%$ of health care costs, then for every $\$ 10,000$ paid by an individual or employer for health care, $\$ 5000$ of that amount goes into the insurance industry.

In provider based insurance, hospitals or groups of doctors essentially agree to treat patients when they get sick for much reduced premiums that are paid directly to the providers themselves. The doctors then form groups to collectively treat patients to ensure that they as a group have the specialist skills and available time to treat the expected volume of patients. A Provider Based Insurance Platform identifies the specialists that must be in the group so that individuals can be treated even they have an illness outside a particular doctor's specialization. In addition it determines the right number and type of specialists required to provide an assurance to treat a particular demographic and geography. To do all this, the platform essentially automates the insurance industry and its actuarial and other functions. This platform is an implementation of the pattern of modelling health care related processes, and then using GCI related adaptive problem-solving patterns to optimize the execution of those processes through automation and other means.

In this way, with this platform, it might be possible to reduce health care costs for the individual, while increasing profitability for doctors. Globally, the implications of this set of platforms are profound, including making medical practices viable in rural and other areas where they might not have been economic before. It's this potential for global impact that may allow this project to attract far greater international cooperation and funding than might be justified by the importance among the international community of the health care needs of any prospective pilot country alone.

Some of the principles of collective intelligence are providing objectively well-defined processes by which the group can cooperate, as opposed to the processes being what some gatekeeper says they are. Other principles are providing a means by which each prospective participant can objectively judge which process optimizes their outcomes, and allowing each participant to decide which process of cooperation to engage in so they can achieve these optimal outcomes. Another is providing a means to objectively rank other group members that are available to cooperate with, where this ranking is according to which of those group members optimizes outcomes of the cooperation. Each participant also needs to be allowed to decide which of those other group members to engage in cooperation with, so that participant can achieve these optimal outcomes for themselves.

In automating the processes of the insurance industry, the Provider Based Insurance Solution 
provides the well-defined processes required for decentralized cooperation. In enabling patients to optimize their outcomes by selecting doctors within these decentralized processes of cooperation, and in enabling doctors to optimize their outcomes by selecting interventions and other means, the Provider Based Insurance Solution and National EHR/Single Virtual Patient Record Solution together can help both patients and doctors cooperate in this collectively intelligent way.

How do you create a healthcare solution that converges on wellness?

In the current healthcare paradigm, incentives are not necessarily aligned to maximize wellness. In a highly litigious nation, a doctor might be most incentivized to give treatments he or she won't get sued for. Or in a less litigious nation, a doctor might be most incentivized to keep the customer coming back. A doctor giving treatments they won't get sued for probably boils down to prescribing medications for which expensive clinical trials have been performed demonstrating the efficacy of the treatment. But the expense of these clinical trials may help ensure that medicines which can't be patented and that don't have a potentially high profit margin, don't get such trials. Is garlic a great treatment for certain ailments? Is ginger? Is chicken soup really good for the common cold? Every single plant on this earth is a medicine in the sense that to varying degrees it produces some effect on this bodily system. Using them as a medicine is a matter of observing the effect and using it intelligently.

This brings me to an anecdote I call "The Forest Told Us". Scientists have long puzzled about how collective learning about natural medicines and potions comes about, some of which require such complex intermediate processing steps it seems unimaginable how, in the absence of scientific knowledge, indigenous peoples could have envisioned the final product from the raw ingredients.

Frank MacEowen, an author of a number of books on spirituality and earth mysticism talks in his book "The Spiral of Memory and Belonging: A
Celtic Path of Soul and Kinship" about how thousands of years ago ancient cultures in the Amazon learned to combine the ingredients required to make a psychedelic substance called ayahuasca. The ingredients aren't psychoactive alone, but together they expose a chemical called DMT or Dimethyltryptamine, commonly known as the spirit molecule, perhaps because people who take it almost universally describe "coming in contact with intelligent beings" during their hallucinations. Unlike other hallucinogenic substances, for some reason DMT anedotally appears to produce such encounters. Westerners who take DMT today commonly describe those beings as "aliens" from another dimension. But the shamans who discovered it generally described them as "spirits" and rather than talking of them coming from another dimension they talked of them coming from the spirit world. Many people, the author says, have asked the shamans how they figured out they needed to combine the different plant materials to make ayahuasca. None of the intermediate products of processing are psychoactive so it is not clear how the processing steps could have been figured out without a knowledge of chemistry. The standard response he says, is "the forest told us".

Looking at this statement metaphorically, there may be profound value in it. It's reasonable to assume that substances which cause the same reactions in our bodies, or in the bodies of other creatures, might contain substances that are similar in some way. Perhaps they are substances that are processed through a similar metabolic pathway. Like the DMT naturally produced in our brain is present in a similar form in some plants. And it's reasonable to assume that whether talking about drying, or boiling with a the bark of a certain tree, processes that change substances in a certain way, might change similar substances in a similar way. Every single plant on earth is a medicine in the sense that it impacts the state of the body in some way, whether large or small. The effects of plants on our bodies, the effects of a given processing step on different plants that create similar reactions within us, and the impact on our bodies of the medicines created through that processing, are all things we can observe. By 
simply observing and being aware of everything that happens around us, and by inferring similarities in our single collective mind, all of these interactions might become clear. In other words, with a single coherent data model that creates the capacity for all observations to contribute to all understanding "the forest will tell us".

Collective intelligence also puts individuals in charge of their own data so that decision-making can be decentralized. By creating a personal repository for personal healthcare data which resides in each patient's sole control, and by creating trusted agents within that repository that can automatically answer queries on behalf of that individual, a collectively intelligent healthcare platform can directly pay individuals to have their personal agent submit anonymized healthcare data in response to queries. By semantically modeling this data so it has human meaning, those agents might answer queries even if they have incomplete data, or if the data is in a different format from that expected by the query. In this way, medical research can begin to conduct massive decentralized studies that can be completed in microseconds rather than in months or years. These studies can allow medical research to observe the effects of all of these potential medicines.

By semantically modeling the processes by which interventions are executed, by predicting the outcomes of each intervention, and by creating a means to objectively compare and select outcomes, collective intelligence can enable simulations to optimize the outcomes of those interventions. Predicting outcomes of each intervention is possible where outcomes are a known function of the intervention. And predicting outcomes of each interventions is possible using pattern detection where there is an observable relationship between intervention and outcome but the mechanism isn't known. And by measuring the difference between predictions and actual results, those predictions can be continuously improved.
Adding decentralization to this semantic data and process storage and capacity for simulations also introduces the possibility of massive decentralized simulation of health and wellness care policy outcomes on a population wide scale. Any simulation might select a representative subset of the population and simulates outcomes of the healthcare policy on those individuals. Rather than having to collect all the individual's data in order to do such a simulation, a decentralized simulation approach might in effect just ask the agent governing each anonymous repository to "simulate the given circumstance" And then it might compile all the answers into a demographic profile for the geography, which it might use to calculate a metric of predicted collective wellbeing for each policy intervention.

The goal in the later phases of the proposed CIPAA-SDGs program is to use these techniques to create platforms that enable the simulation of not only individual interventions to help doctors select the best ones, but also to simulate entire healthcare and other social policies to select optimals ones.

Using all of these techniques a collective intelligence approach might potentially have a transformative impact on healthcare. Beginning in phase II, the proposed CIPAA-SDGs program aims to deploy these healthcare solutions with the potential to save enough in healthcare cost to pay for universal health coverage for those currently without. Beginning in phase III, the proposed CIPAA-SDGs program aims to deploy massively collaborative decentralized medical querying solutions with the potential to conduct surveys from stored data in microseconds rather than months. Beginning in phase IV the proposed CIPAA-SDGs program aims to deploy these collective intelligence platforms to simulate outcomes of healthcare policies to optimize policymaking, and to simulate outcomes of medical interventions to optimize the practice of medicine, including perhaps collectively observing the pharmacological impacts of new substances. 
The collective intelligence infrastructure the proposed CIPAA-SDGs program will build in its earlier phases [18] is intended to make all of this possible.

\section{Conclusions}

This paper explores two proposed applications of GCI to healthcare. One is the application of GCI to the design of software platforms including an Electronic Health Record (EHR) and an Providerbased Health Insurance platform, where an assessment of the combined savings to the health system due to that collectively intelligent cooperation suggests the potential savings to be sufficient to pay for universal healthcare for those

\section{Acknowledgements.}

Thanks to Julian Talev for countless discussions on the topic.

\section{References}

1 Journal article: Mahase Elisabeth. Covid19: What is the evidence for cloth masks? BMJ 2020; 369 :m1422

2 Pre-print: Williams, A. E. (2020, April 20). The Global Response to COVID-19 as an Example of a One-Sided Problem Definition in the Absence of General Collective Intelligence. https://doi.org/10.31234/osf.io/emgxc

3 Pre-print: Williams, A. E. (2020, May 15). Use of Human-Centric Functional modelling to Maximize Convergence in Integrative Research. https://doi.org/10.31730/osf.io/jv6h8

4 Pre-print: Williams, A. E. (2020, April 16). A Human-Centric Functional modelling Framework for Defining and Comparing Models of Consciousness and Cognition. https://doi.org/10.31234/osf.io/94gw3

5 Book Chapter: Williams A.E. (2020) A Model for Artificial General Intelligence. In: Goertzel B., Panov A., Potapov A., Yampolskiy R. (eds) Artificial General Intelligence. AGI 2020. Lecture Notes in without coverage. The second is the use of the functional state spaces as tools for making clinical observations. Because every plant on earth is a potential medicine in that it has some effect on the human system. However observing those impacts is difficult with the unstructured and incomplete data generally available outside of targeted research. Defining the human system in terms of unambiguous functional state spaces, and defining medicines and other interventions as transforms in those state spaces is proposed to enable observations made in different systems of medicine to be comparable in common terms, so they can be combined into a single database in which a medical study to deduce the impact of any intervention is simply a slice of the data.

Computer Science, vol 12177. Springer, Cham. https://doi.org/10.1007/978-3-03052152-3 38

6 Pre-print: Williams, A. E. (2020, December 2). General Collective Intelligence vs the Innate Collective Intelligence Factor. https://doi.org/10.31730/osf.io/kp3x8

7 Conference: The Relationship Between Collective Intelligence and One Model of General Collective Intelligence, Andy E. Williams, Computational Collective Intelligence, 11th International Conference, ICCCI 2019, Hendaye, France, September 4-6, 2019, Proceedings, Part II, Pages 589600

8 Journal article: Woolley, Anita Williams; Chabris, Christopher F.; Pentland, Alex; Hashmi, Nada; Malone, Thomas W. (29 October 2010). "Evidence for a Collective Intelligence Factor in the Performance of Human Groups". Science. 330 (6004): 686$688 . \quad$ Bibcode:2010Sci...330..686W. doi:10.1126/science.1193147. PMID 20929725. S2CID 74579.

9 Journal article: Krafft, P.M. (2019), A Simple Computational Theory of General Collective Intelligence. Top Cogn Sci, 11: 374-392. https://doi.org/10.1111/tops.12341. 
10 Book: Malone, T. W. (2018). Superminds: The surprising power of people and computers thinking together.

11 Journal article: Williams, Andy E. "A Model for Human, Artificial \& Collective Consciousness (Part I)." Journal of Consciousness Exploration \& Research 10.4 (2019).

12 Journal article: Williams, Andy E. "A Model for Human, Artificial \& Collective Consciousness (Part II)." Journal of Consciousness Exploration \& Research 10.4 (2019).

13 Working Paper: Andy E. Williams, Defining a Sensory State Space as the Functional State Space of the Body, 2020.

14 Working Paper: Andy E. Williams, Detecting Truth in Functional State Space with Artificial General Intelligence and General Collective Intelligence, 2020.

15 Book: Kahneman, Daniel. Thinking, Fast and Slow. New York: Farrar, Straus and Giroux, 2011.

16 Pre-print: Williams, A. E. (2020, December 16). Individualization of Products and Services with Artificial General Intelligence and General Collective Intelligence. https://doi.org/10.31730/osf.io/ gd5mt

17 Pre-print: Williams, A. E. (2020, November 17). Bridging the Left - Right Political Divide with Artificial Intelligence and Collective Intelligence. https://doi.org/10.31730/osf.io/y65x4

18 Pre-print: Williams, A. E. (n.d.). The Peer to Peer Social Fabric as a Platform for General Collective Intelligence. Retrieved from osf.io/preprints/africarxiv/qbxfr

19 Pre-print: Andy E. Williams, Discovering and Implementing Self-Sustaining Networks of Cooperation with General Collective Intelligence, working draft (2020) 